\title{
Nuevos planteamientos didácticos: ¿Al innovar en docencia, mejoramos el aprendizaje?
}

\author{
¿Cuándo innovamos en docencia, \\ mejoramos el aprendizaje? \\ A. Cornet Calveras \\ Universitat de Barcelona
}

En primer lugar: ¿Qué entendemos por innovación docente? Desde un aspecto amplio de su definición hemos de entender la innovación como "el arte de aplicar, en condiciones nuevas, en un contexto concreto y con un objetivo preciso las herramientas técnicas, científicas, metodológicas, a nuestro alcance".

En este sentido, los procesos de innovación educativa han de contemplar no sólo aquellos aspectos de investigación sobre nuevos procedimientos, metodologías o tecnologías, sino también, y especialmente, la asimilación de aquellos aspectos ya desarrollados, dominados y aplicados con éxito en otros campos de actividad, pero que constituyen una novedad en su aplicación en el contexto de los procesos de enseñanza-aprendizaje

¿La innovación ha ayudado a mejorar la docencia tradicional?

Centrándonos en el caso de educación superior, la metodología tradicionalmente utilizada en la docencia universitaria ha sido magistral, teniendo en cuenta que este concepto no sólo describe el tipo de actividad docente sino que también una manera de entender el aprendizaje como un proceso en el cual la formación del estudiante pasa por proporcionarle unos conocimientos que él ha de asimilar. Dentro de este contexto, los procesos de innovación consistentes en la incorporación de nuevos materiales, introducción de nuevas metodologías y prácticas en la docencia han introducido, en la mayoría de los casos, mejoras en los procesos de enseñanza-aprendizaje.

Por ello, hemos de dar una respuesta afirmativa a la pregunta.

¿Qué papel ha de jugar la innovación en el futuro inmediato?

Actualmente, las Universidades se enfrentan al reto de adaptar sus procesos de formación a las exigencias de los cambios radicales que se producen en su entorno:

Los cambios propiciados por la denominada sociedad de la información, en la cual aparte de proporcionar conocimiento se ha de formar al estudiante en la gestión y utilización de la gran cantidad de información disponible.

Los cambios en el perfil de los estudiantes (más heterogéneos, compatibilidad con el trabajo,...)

Los cambios producidos por los avances científicos, que requieren una actitud crítica y abierta

Los cambios propiciados por la incorporación de las TIC a la vida cotidiana de los futuros estudiantes/usuarios de las universidades.

Una sociedad más compleja, más diversa, más libre, que necesita ciudadanos formados e informados que sepan enfrentarse a situaciones cambiantes y complejas.

A esta situación se añaden las exigencias impuestas por el proceso de adaptación al espacio europeo de educación superior, que modifican radicalmente el panorama universitario.

Por ello, las instituciones universitarias han de adaptar los pro- cesos de enseñanza-aprendizaje con el objetivo de acentuar la implicación activa del estudiante en su proceso de aprendizaje, dando énfasis a la adquisición tanto de conocimientos como de habilidades, preparando al estudiante para asumir responsabilidades en un mundo en rápido y constante cambio, mejorando sus capacidades emocionales e intelectuales y dotándoles de las herramientas necesarias para que puedan enfrentarse a las exigencias de un procedimiento de formación continuada a lo largo de su vida.

En este contexto, los métodos tradicionales siguen siendo necesarios, pero no suficientes para conseguir estos objetivos.

Por ello, es indiscutible la necesidad de introducir procesos de innovación docente para mejorar el aprendizaje.

Sin embargo, la innovación educativa es un proceso complejo en el cual se han de implicar no solamente el profesorado a nivel individual, sino también la institución de manera que los cambios introducidos se hagan de acuerdo con un planteamiento bien establecido y siguiendo una estrategia planeada de manera sistematizada e intencionada de acuerdo con la consecución de los objetivos propuestos.

Siguiendo este razonamiento, la respuesta tiene que ser obvia Innovando la docencia, mejoramos el aprendizaje.

\section{Cambio de paradigma docente de la Escuela de Enfermería Vall d'Hebron de Barcelona: enseñanza tradicional versus aprendizaje basado en problemas M. Cònsul Giribet}

Escuela Universitaria de Enfermería Vall d'Hebron

Después del proceso de evaluación interna y externa, los docentes de la Escuela de Enfermería Vall d'Hebron, nos vimos con la responsabilidad y el entusiasmo suficientes para afrontar un cambio de metodología más acorde con las nuevas demandas sociales y profesionales. El reto que nos planteábamos era de una transformación profunda, y nuestro interés se centró en la metodología del Aprendizaje Basado en Problemas (ABP) porqué se basa en un paradigma docente centrado en el aprendizaje del estudiante y no en la enseñanza del profesor; porqué potencia más las competencias que el estudiante tiene que adquirir, y también porqué hay una formulación más concreta de los objetivos en lugar de los programes de las asignaturas del Plan de Estudios.

El cambio lo iniciamos con una experiencia piloto el curso 2001-2002 y de la mano del profesor Luis Alberto Branda que llevaba aplicando esta metodología desde que se inició el 1969 en la Universidad de McMaster en Canadá. En el curso siguiente 2002-2003 iniciamos el cambio global a primer curso, y actualmente tenemos implantada la nueva metodología a los tres cursos académicos de la Diplomatura de Enfermería.

En el ABP, al trabajar con grupos reducidos de 8-10 estudiantes, permite el proceso de reconstrucción de su conocimiento mediante procesos de diálogo y discusión que les ayuda a desarro- 
llar habilidades transversales de comunicación y expresión oral; de pensamiento para la formulación de juicios; de argumentación lógica y para la defensa de sus valores y puntos de vista, que les ha de permitir afrontar una practica profesional con un pensamiento más crítico.

Con un aprendizaje integrador, como lo es el ABP, se pretende que las experiencias de salud se vean desde todas les vertientes (biológicas, psicológicas, sociales y culturales...). Al no fragmentar, hay una cantidad extrema de interacciones e interferencias que permiten al estudiante experimentar la incertidumbre que supone ver el problema desde las diferentes magnitudes, y con la necesidad de buscar el conocimiento que le ha de permitir aproximarse a la complejidad del problema. Con una metodología por problemas, se experimenta el aprendizaje de la misma manera que lo hará en la práctica profesional: contextualizando e integrando los saberes.

EL DESAPRENDIZAJE DE LOS DOCENTES

$\mathrm{Al}$ integrar asignaturas el profesorado hemos tenido que compartir miradas, culturas, contradicciones, objetivos, creencias y pensamientos que, al confrontarse, nos ha permitido trabajar con la lógica de la complejidad. Edgar Morin (2001) dice que la complejidad está animada por una tensión permanente entre la aspiración a un saber no parcelado, no dividido, no reduccionista, y el reconocimiento de que todo conocimiento es incompleto e inacabado.

La coherencia del propio método ABP nos ha aportado a los docentes una nueva cultura de trabajo en equipo y un clima de confianza mutua para compartir dudas, problemas y angustias, que quizás no percibíamos tanto con la enseñanza tradicional más anclada en la cultura del individualismo.

Trabajar cinco años con esta metodología me ha dado elementos para afirmar que es una herramienta útil para la formación de los futuros profesionales que se tendrán que enfrentar al vertiginoso ritmo de los avances científicos y tecnológicos, y a los problemas de esta nueva civilización llamada de la información, de la comunicación y del conocimiento. Todos sabemos la cantidad de conocimiento que se genera en todas las disciplinas y particularmente en las de ciencias de la salud que lo hace de manera exponencial. Para gestionar este problema hemos de enfatizar en desarrollar habilidades para "aprender a aprender", para permitirle un auto-reciclaje a lo largo de su vida profesional.

La adopción de la metodología $\mathrm{ABP}$ a los estudios de enfermería es un reto pera los docentes y para la propia profesión de enfermería. El ABP nos brinda la oportunidad de aprovechar un aprendizaje participativo para promover la reflexión, cuestionar permanentemente el conocimiento, y formar profesionales críticos, con habilidades y competencias que les ha de permitir ser más autónomos y proporcionar curas eficientes, efectivas y humanas, en una realidad cargada de incertidumbre y complejidad.

Bibliografia:

Branda, L. A. (2001) Innovaciones educativas en Enfermería: Aprendizaje Basado en Problemas, centrado en el estudiante y en grupos pequeños. Revista Rol de Enfermería. 2001; 24(4)

Dewey, J. (1993) Como pensamos: Nueva exposición de la relación entre pensamiento reflexivo y proceso educativo. Barcelona: Cognición y desarrollo humano. Paidos

Morin, E. (2001) Tenir el cap clar: per organitzar els coneixements i aprendre a viure. Barcelona: La Campana

Schön D. (1987) La formación de profesionales reflexivos: Hacia un nuevo diseño de la enseñanza y el aprendizaje en las profesiones. Barcelona: Paidos

\section{El Powerpoint: del uso al abuso J. Palazón Barandela}

Unitat de Fisiología Vegetal.

Facultat de Farmacia. Universitat de Barcelona

La enseñanza es el principal proceso intencional mediante el cual la sociedad moderna convierte a sus individuos en herederos de su saber. (Flórez, 1994)

La cultura de la sociedad de la información, asentada en el principio de globalización cultural y económica, y en los constantes avances científico-tecnológicos ha presionado con fuerza en todos los estamentos universitarios, conjuntamente con las particularidades específicas de nuestro contexto más próximo (baja tasa de natalidad, papel específico de la unión Europea, punto de convergencia de culturas,...). El resultado es una evolución cada vez más acelerada de la Universidad para adaptarse al cambiante entorno social, que supone un replanteamiento de su propia razón de ser, de sus objetivos y servicios, de los métodos e instrumentos de trabajo, de planes de estudio, etc. En este contexto cambiante, las Tecnologías de la Información y la Comunicación (TIC) pueden aportar grandes ventajas en los cuatro ámbitos principales de la actividad universitaria: docencia, investigación, gestión y presencia en el entorno social.

Disponer de nuevos recursos que puedan permitir nuevas formas de proceder no significa que necesariamente se produzca un cambio en la Universidad, sobre todo a nivel docente. Antes el profesor daba sus clases magistrales con el apoyo de la pizarra y los alumnos presentaban sus trabajos y exámenes escritos a mano o máquina; ahora el profesor da sus clases magistrales con "PowerPoint", los estudiantes presentan sus trabajos en "Word" y a veces, los exámenes son pruebas objetivas ante un ordenador. ¿Dónde está el cambio? ¿Innovación o simple comodidad? La disponibilidad de las TIC por parte de profesores y estudiantes no supone el fin de los aprendizajes basados en la memorización y la reproducción de los contenidos, pero hay aspectos que obligadamente han cambiado como es la mayor universalización de la información; ahora el problema de los estudiantes ya no es el acceso a la información sino la aplicación de metodologías para la búsqueda inteligente y crítica de la misma. Este hecho nos remite a revisar el concepto de "aula de clase" con el propósito central de superar la condición de profesores intuitivos e informadores por la de científicos, formadores y responsables de la calidad de la enseñanza.

Damaris, en 1999, daba una serie de recomendaciones para mejorar la docencia como son: prestar menos atención a la lección magistral y más atención al asesoramiento que facilite el aprendizaje de nuestros alumnos, tratar de evitar la docencia en aulas masificadas y dejar de dar prioridad a la comunicación exclusivamente verbal para integrar la comunicación verbal con la comunicación audiovisual en las presentaciones didácticas. De todas estas recomendaciones, puede ser esta última, la seguida en mayor proporción por el Profesorado Universitario, con la incorporación del PowerPoint como sistema audiovisual, al impartir nuestras clases magistrales. Microsoft PowerPoint es un programa orientado a diseñar presentaciones gráficas. Incorpora todas las herramientas gráficas para crear de forma fácil y sencilla presentaciones de apariencia profesional. Nos permite crear rápidamente transparencias, diapositivas o efectos sorprendentes para hacer una presentación en pantalla y la creación de vínculos que, a través de 
Internet nos pueden acercar a conferencias impartidas por especialistas en cualquier lugar del planeta, páginas web, publicaciones generales o específicas, etc.

La utilización correcta del PowerPoint en el aula puede aportar innumerables ventajas a la enseñanza, presentaciones más ordenadas y vistosas, con las que captar mejor la atención de nuestros alumnos, esquemas más cercanos a la realidad de los procesos, destacar e ilustrar los aspectos más relevantes de nuestras explicaciones, etc. Pero muchas veces el docente aprovechando sus ventajas, lo utiliza de forma inadecuada, cargando la pantalla de contenidos, para evitar tener que memorizarlos, imprimiendo un ritmo a la clase propio de una conferencia, más que de una lección magistral, tratando de mantener la atención del alumno simultáneamente en numerosos puntos como son, la explicación hablada, la pantalla donde se proyecta y la pizarra. Si a estos factores se le une una incorrecta utilización técnica de esta herramienta, utilizando fondos poco contrastados, defectos de iluminación en el aula, exceso de movimientos en las presentaciones, se puede generar un ambiente hostil que acabe provocando aquello que el docente pretende evitar, que el alumno abandone el aula, hastiado y sintiéndose incapaz de asimilar los conocimientos que su profesor ha pretendido transmitirle durante la clase, desvirtuándose por completo el proceso docente.

Bibliografía:

Flórez, R. 1994. Hacia una pedagogía del conocimiento. Ed. McGraw-Hill

Damaris, H. 1999. La didáctica Universitaria. Referencia imprescindible para la enseñanza de calidad. Revista Electrónica Interuniversitaria de Formación del Profesorado. 2(1).

[<http://w.w.w.uva.es/aufop/publica/revelfop/99-v2n1.htm>]

\section{Los nuevos retos de la educación superior. J. Mateo}

Facultad de Pedagogía. Universitat de Barcelona.

El nacimiento del milenio ha incrementado nuestra sensibilidad ante las nuevas realidades que se están generando que hacen más patente nuestra desorientación e incapacidad para construir respuestas significativas antes sus desafíos.

No estamos viviendo, como en otras ocasiones, una de las periódicas crisis coyunturales a que nos tiene acostumbrado el modelo social vigente de desarrollo, los cambios actuales se sitúan en otro nivel y se substantivan con la aparición de nuevas formas de organización social, económica y política y con las transformaciones de todo orden que las acompañan.

Vivimos la inquietante sensación que el mundo cambia más rápidamente que nuestra capacidad organizativa para adaptarnos a él. En la base del cambio se sitúan fenómenos complejos e inter-relacionados que adoptan en este espacio de modernidad nuevos significados.

Así tenemos la revolución digital, el continuo crecimiento y la rápida obsolescencia de la información y del conocimiento, la globalización y la nueva organización del trabajo entre otros, incrementan de forma continua y acelerada la complejidad de la sociedad y nos plantean con urgencia la necesidad de nuevas interpretaciones de la realidad buscando en las instituciones educativas, especialmente en las universitarias, señales para la creación de nuevos discurso que nos ayuden a comprenderla y a manejarnos más eficientemente dentro de ella.
En este contexto la institución universitaria se encuentra a su vez, en plena crisis de cambio y es consciente, posiblemente por primera vez en su larga historia, que su pervivencia, al menos con el estatus que actualmente disfruta, depende de su capacidad de asumir con decisión, imaginación y coraje todas las transformaciones que precisa para favorecer su propia y absolutamente necesaria transformación.

\section{La innovación en docencia.}

Si nos centramos en el objeto de esta mesa redonda y tratamos de concretar nuestra aportación en lo que hace referencia a la innovación en docencia y siendo plenamente conscientes de los elementos planteados en el apartado anterior, no podemos orientar la innovación hacia acciones de puro maquillaje del sistema. Innovar, por sí mismo, no supone la solución a la crisis, es fundamental captar la verdadera naturaleza y sentido del cambio en este apartado si no queremos caer en actuaciones perversas enmascaradoras de los verdaderos problemas.

El proceso de enseñanza-aprendizaje constituye posiblemente uno de los ejes básicos sobre los que necesariamente habrá que construir los nuevos espacios de modernidad de la Universidad y entendemos que la innovación en este apartado se debería centrar sobre cuatro apartados básicos.

Cambios en el enfoque del proceso docente: del énfasis en la enseñanza al énfasis en el aprendizaje.

Uno de los cambios fundamentales a realizar es respecto del enfoque clásico del binomio enseñanza-aprendizaje. La tendencia es centrar la acción en los resultados de los aprendizajes en detrimiento de los medios en términos de recursos que se han invertido para conseguirlo.

Lo importante es desarrollar la capacidad del estudiante para aprender ahora y a lo largo de toda la vida. La enseñanza no es, sino un medio para conseguirlo. El buen profesor es quien mejor gestiona los escenarios y los medios para lograr el aprendizaje de sus alumnos.

El propio enfoque actual de los ECTS, coloca su centro de gravedad en la gestión de las actividades de aprendizaje de los estudiantes.

Cambios en la naturaleza de los contenidos de aprendizaje.

La naturaleza de las actividades de aprendizaje se modifican sustancialmente, ampliando el campo de contenidos y asignándoles y enfatizando sus valores de forma distinta a la tradicional.

Así a los contenidos académicos clásicos se añaden los contenidos procedimentales y actitudinales y a todos ellos se les complementa con un conjunto de capacidades y habilidades de carácter transversal que también deberían ser objeto de aprendizaje y evaluación.

De lo que se trata ya no es de aprender muchas cosas sino de desarrollar la capacidad de hacer un uso competencial de lo que necesitamos saber para resolver problemas y situaciones de la vida real. El aprendizaje se programa para su continuidad a lo largo de toda la vida. Nuestra tarea fundamental como profesores es desarrollar en nuestros alumnos las competencias necesarias para actuar de acuerdo a estos principios.

Cambios en la lógica de la evaluación de los aprendizajes.

En este contexto es donde la evaluación de los aprendizajes cobra una especial dimensión y focaliza su atención no tanto en controlar y acreditar lo aprendido (que también) sino incidir en la mejora de los aprendizajes, especialmente desarrollando la capacidad autónoma de los estudiantes para aprender. 
Es obvio que si le asignamos a la evaluación este nuevo papel, no podemos anclarnos en los modelos clásicos de evaluación. A las pruebas tradicionales habrá que incorporar un conjunto de nuevas estrategias evaluativas, más centradas en valorar y mejorar las propias ejecuciones que en juzgar lo aprendido

de manera formal.

Cambios en la gestión del aprendizaje.

Como consecuencia de los otros tres apartados, nos enfrentamos a un replanteamiento en profundidad del modelo de gestión de los aprendizajes. Este nuevo modelo deberá contemplar simultáneamente los factores siguientes:

- Una concepción de la docencia basada en una visión integral del proceso de enseñanzaaprendizaje y donde no se programará por temarios sino por actividades de carácter comprensivo e integrador.

- Un enfoque metodológico fundamentado en la adquisición del conocimiento por interacción con las realidades donde se aplica.

- Un sistema de evaluación basado en el análisis reflexivo y crítico de ejecuciones de actividades próximas a la propia realidad.

A modo de síntesis.

Para finalizar señalamos los puntos que consideramos más básicos de nuestra intervención:

- Tendremos que entender el binomio enseñanza-aprendizaje de forma absolutamente integrada. Constituyen las dos caras del mismo proceso. En todo caso el elemento nuclear es el aprendizaje.
- La docencia entendida como planificación estratégica se tendrá que diseñar desde las necesidades surgidas de los aprendizajes que han de lograr los estudiantes.

- El conocimiento se tendrá que tratar de forma comprensiva y no fragmentada. Es necesario establecer las relaciones más pertinentes dentro y fuera de la propia área de conocimiento. Hemos de hacer especial énfasis en que los alumnos desarrollen plena consciencia respecto de lo que saben.

- Metodológicamente estos nuevos enfoques obligarán a nuevas formas de programación estructuradas no tan sólo respecto de los contenidos a aprender sino también de las actividades a desarrollar. Cada actividad ha de justificar los conocimientos y habilidades que precisa y las competencias que pretende desarrollar.

- La evaluación pasará a constituir un elemento fundamental de esta nueva metodología. Necesitaremos generar nuevas prácticas evaluativas que, de forma complementaria a las ya conocidas, favorezcan la reflexión y la consciencia respecto de lo que estamos aprendiendo y detecten con seguridad las deficiencias del propio proceso.

Finalmente hemos de señalar que será preciso instaurar una nueva cultura docente e institucional. Sin transformación personal, profesional y colectiva es del todo imposible instalarse en una dinámica productiva y coherente de cambio. 